\title{
Composition, morphology and crystallinity biomaterials based on calcium phosphate for implant bone regeneration
}

\section{P-BR 052}

\section{BASIC RESEARCH}

Zenobio Elton ${ }^{1 \star}$, Cosso Mauricio', Nogueira Maria, Abreu Fernando', Antunes Alberto', Santiago Julia², Zenobio Madelon ${ }^{3}$

'Associate professor, Department of Dentistry, Pontifícia Universidade Católica de Minas Gerais, Belo Horizonte, Minas Gerais, Brazil

Master's degree program in Dentistry, Department of Dentistry, Pontifícia Universidade Católica de Minas Gerais, Belo Horizonte, Minas Gerais, Brazil

${ }^{3}$ Researchers of CDTN Nuclear and Technology Development Center, Belo Horizonte, Minas Gerais, Brazil

\section{Background and Aim}

The alveolar bone and surrounding soft tissues must present volume and ideal qualities in order to receive implants. When these characteristics are lacking, biomaterials based on $\mathrm{Ca}$ and PO4 composition are regularly used to obtain adequate bone volume and height. The inorganic phase of bones and teeth is characterized by a dominant concentration of calcium phosphate. This has led researchers to develop biomaterials with similar chemical composition and characteristics. The present study analyzed the chemical composition, morphological aspect and crystallinity of four biomaterials, Bio-Oss, Cerasorb, BoneCeramic and Osteogen.

Material and Methods

The analyses applied were instrumental neutron activation (NAA), energy dispersive X-ray (DX), elemental analysis (EA), X-ray diffraction (XRD), thermogravimetric (TGA), scanning electron microscopy (SEM) and energy dispersive spectroscopy (SEM/EDS).

\section{Results}

The chemical analyses were effective and detected differences in the elemental composition between the studied biomaterials. The most highly concentrated elements in the biomaterials based on calcium phosphate were $\mathrm{Ca}$ and $\mathrm{P}$. Other elements (Al, K, V, Mn, Br, La, Sm, Eu, W, Na, Mg, Si, S, Cl, Fe, Zn, Sr) that are no usually found in the inorganic bone structure were also detected. Thermogravimetric curves agree with the data of the elemental analysis of $\mathrm{C}, \mathrm{H}$ and $\mathrm{N}$, regarding the content of organic groups, however in Cerasorb\&\#61666; no data could be identified due to the lack of organic elements. Osteogen and Boneceramic, perhaps are synthetic elaboration, presented a low carbon content $(0.23 \%$ and $0.12 \%)$. Morphological data assessed using SEM revealed that these four biomaterials are quite different in shape, surface topography and crystalline size.

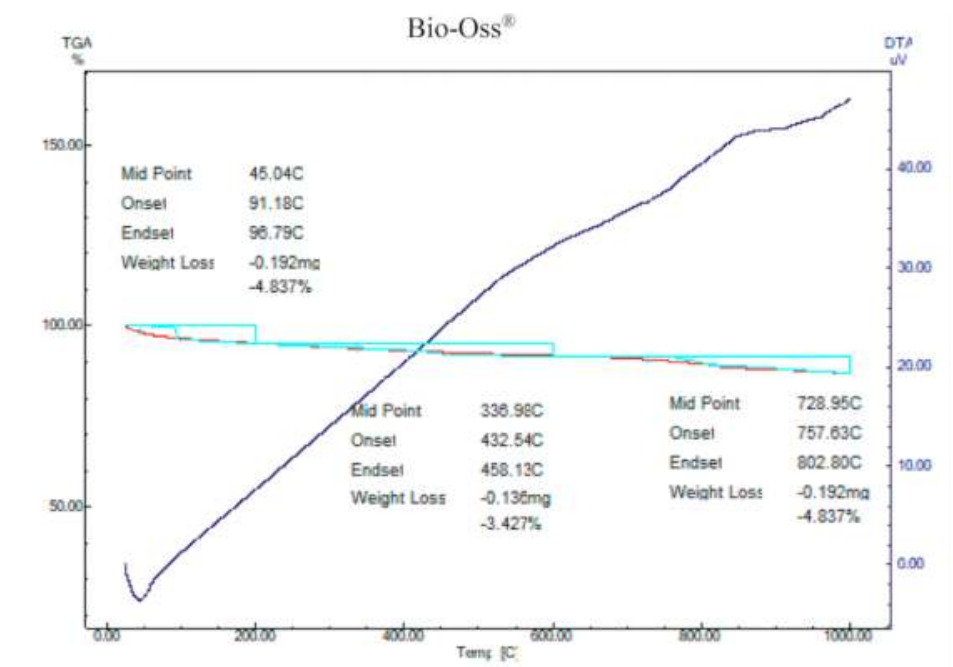

Figure 1 - TG- Bio-OSS ${ }^{\circledR}$

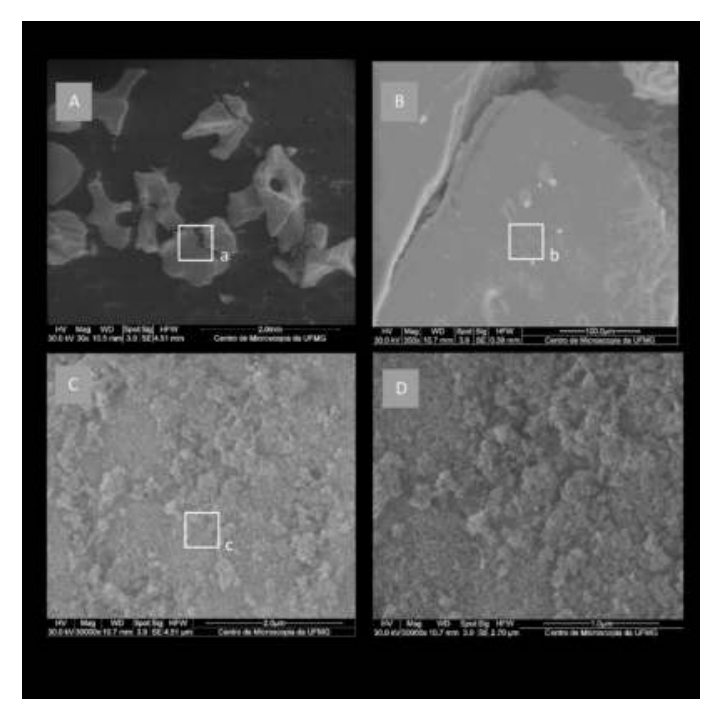

Figure 4 - Scanning electronic microscope image of Bio-Oss - 30x. Particle shape is irregular, with welldefined edges.

- 350x. Represents the area displayed in (a). The irregularity of the surface could not $-30,000 x$

(b) The surfacesents the area displayed - 50,000x. Represents the area displaye in (c). At this magnification, it is possible to observe rougher surface.

\section{CONCLUSION}

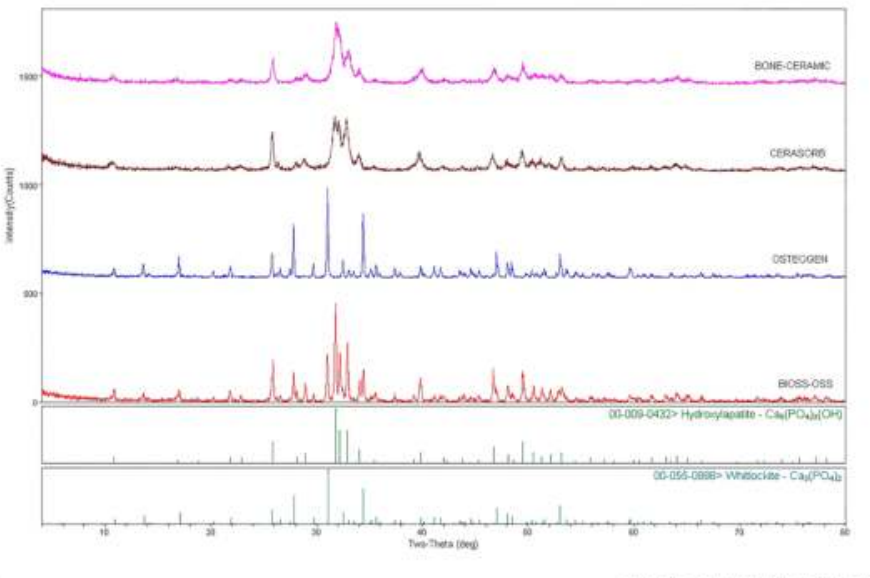

Figure 2 - Results of analysis X-ray diffraction

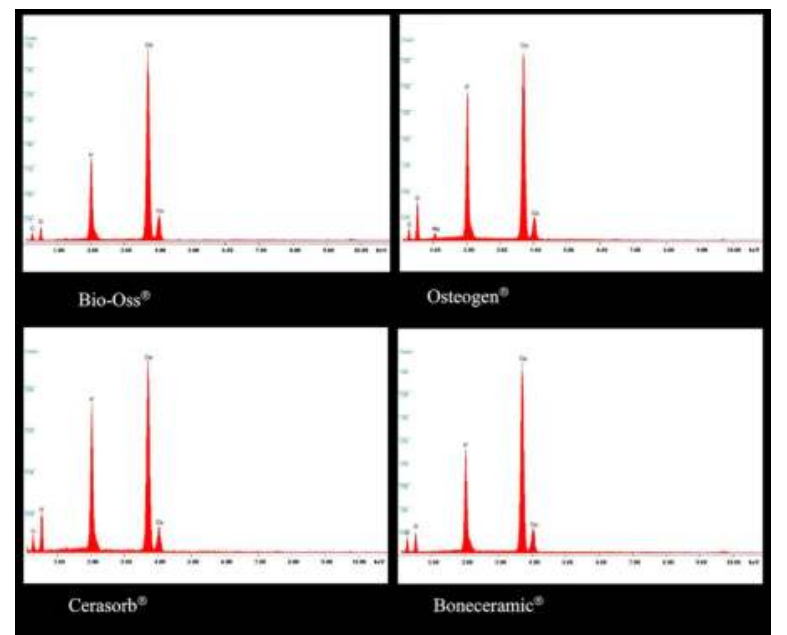

Figure 3 - EDS and crystal sizes of the studied biomaterials

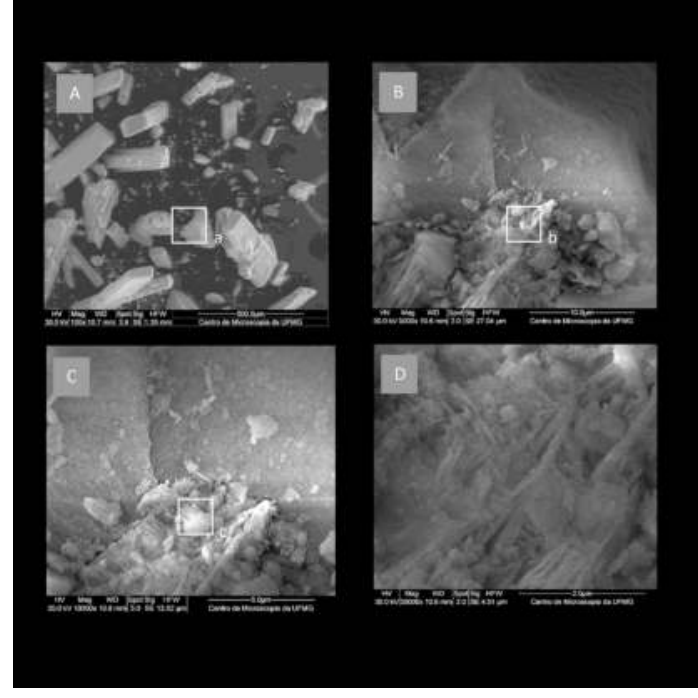

Figure 5 - Scanning electronic microscope

image of Osteogen ${ }^{\oplus}$.
A - 30x. Shows the particles with more compact and a dense surface appearance. B - 5000x. Represents the area displayed in (a). It is possible to note that there is part of the particle with more regular appearance and a portion covered with some irregular thinner small particles like represed in the area with ( (b). It is posible to the an displayed in (b). It is possible to see an area with shows the same aspect as the 5000x, threadlike filaments.

D - 30,000x. Represents the area displayed in (c). At this magnification, it is possible observe irregular and $r$

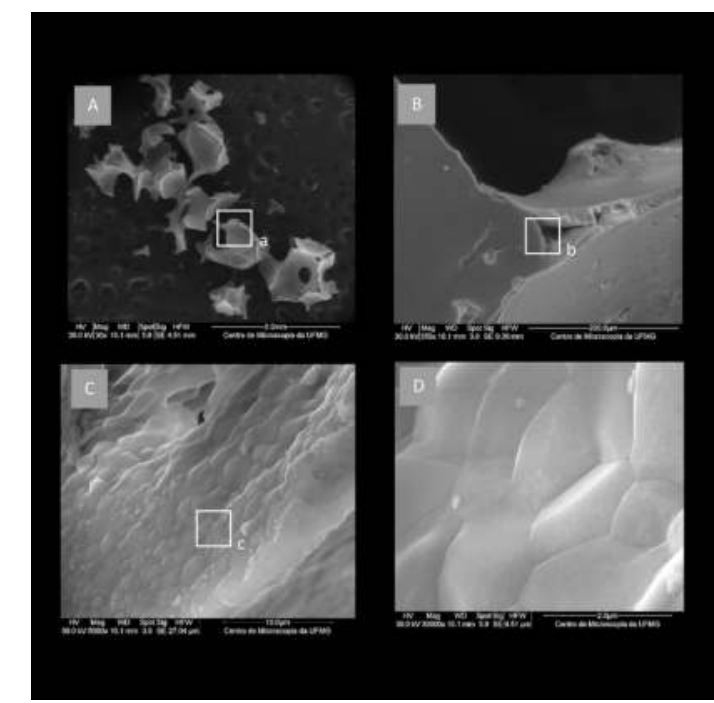

Figure 6 - Scanning electronic microscope

列 sharpened edges.

B - 350x. Represents the area displayed in smooth appearance and an irregular edge. It's possible to identify a gap (b).

C - 5000x. Represents the area displayed in (b). It is possible to observe the surface of the particle with roughness. This roughness is maintained deep in the entire gap ( $)$. $D-30,000 x$. Represents the area displayed in $\left({ }^{*}\right)$. It's shows a smoother surface of the
particle, having a slightly rippled characteristic.

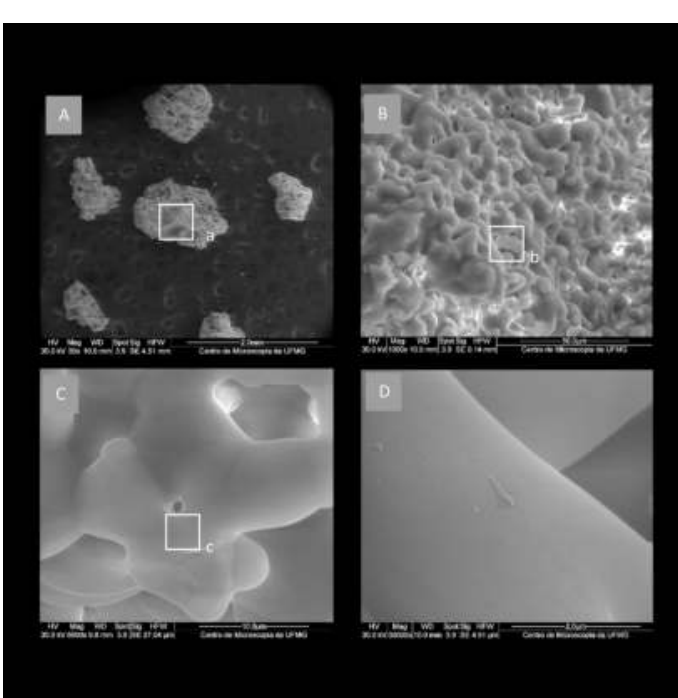

Figure 7 - Scanning electronic microscope image of Cerasorb

A - 30x Shows the biomaterial particles with irregular surface, without a defined shape. (a). In this region, there is a rough appearance, with bullous prominences. C - 5000x. Represents the area displayed in (b). It is possible to observe a smooth surface with some small protrusions and depressions. D - 30,000x. It's shows in detail the smooth surface of the material.

The biomaterial features may determine their biological, biochemical and biomechanical performance during bone formation and, consequently, their successful clinical use

\section{REFERENCES}

Legeros RZ. Formation and transformation of calcium phosphates: relevance to vascular calcification. Z Kardiol 2001; (90):116-124.

Legeros RZ, Lin S, Rohanizadeh R, Mijares D, Legeros JP. Biphasic calcium phosphate bioceramics: preparation, properties and applications. J Mater Sci: Mater in Med. 2003; (14):201-209

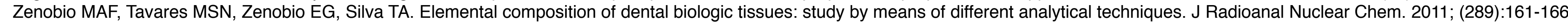

